\title{
Strong Proofs of Knowledge
}

\author{
Oded Goldreich
}

\begin{abstract}
The concept of proofs-of-knowledge, introduced in the seminal paper of Goldwasser, Micali and Rackoff, plays a central role in various cryptographic applications. An adequate formulation, which enables modular applications of proofs of knowledge inside other protocols, was presented by Bellare and Goldreich. However, this formulation depends in an essential way on the notion of expected (rather than worst-case) running-time. Here we present a seemingly more restricted notion that maintains the main feature of the prior definition while referring only to machines that run in strict probabilistic polynomial-time (rather than to expected polynomial-time).
\end{abstract}

Keywords: Proof of Knowledge, Zero-Knowledge

This work was completed in May 1998, and was integrated in the author's work Foundation of Cryptography as [7, Sec. 4.7.6]. The current revision is intentionally minimal.

\section{Introduction}

The reader is referred to [3] for a discussion of the intuitive notion of a proof-ofknowledge (cf., [11]), and the previous attempts to define it $[4,13]$, cumlinating in the definition presented in [3]. We also assume that the reader is familiar with the definition given in [3].

The definition given in [3] relies in a fundamental way on the notion of $e x-$ pected running-time. Throughout the years we remained bothered by this feature, and while working on [6] we decided to look for an alternative. Specifically, we present a more stringent definition in which the knowledge extractor is required to run in strict polynomial-time (rather than in expected polynomial-time). We call proof systems for which this more stringent definition holds, strong proofs of knowledge (in contrast to ordinary proofs of knowledge as defined in [3]).

There are two reasons to prefer strong proofs of knowledge over ordinary ones. Firstly, we feel more comfortable with the notion of strict polynomial-time than with the notion of expected polynomial-time. For example, it is intuitively unclear why a machine which runs for time $2^{n}$ on an $2^{-n}$ fraction of its coin-tosses (and in linear time otherwise) should be considered fundamentally different than a machine which runs for time $2^{n^{2}}$ on the same fraction. Secondly, it seems much more convinient to work (i.e., to compose) strict polynomial-time computations rather than expected polynomial-time ones. (For further discussion of this issue, the interested reader is directed to [9].) 
Unfortunately, there seems to be a loss in going from ordinary proofs of knowledge to strong ones: Not all proofs of knowledge are known to be strong proofs of knowledge. Furthermore, we conjecture that there are proofs of knowledge that are not strong proofs of knowledge (see Section 4). Still, zero-knowledge strong-proofs-of-knowledge do exist for all NP-relations, provided that one-way functions exist.

\section{The Definition}

We assume that the reader is familiar with the definition of a proof of knowledge (as presented in [3]) as well as with the underlying motivation.

Definition 1 (System of strong proofs of knowledge): Let $R$ be a binary relation. We say that an efficient strategy $V$ is a strong knowledge verifier for the relation $R$ if the following two conditions hold.

- Non-triviality: There exists an interactive machine $P$ such that for every $(x, y) \in R$ all possible interactions of $V$ with $P$ on common-input $x$ and auxiliary-input $y$ are accepting.

- Strong Validity: There exists a negligible function $\mu: \mathbb{N} \mapsto[0,1]$ and a probabilistic (strict) polynomial-time oracle machine $K$ such that for every strategy $P$ and every $x, y, r \in\{0,1\}^{*}$, machine $K$ satisfies the following condition:

Let $P_{x, y, r}$ be a prover strategy, in which the common input $x$, auxiliary input $y$ and random-coin sequence $r$ have been fixed, and denote by $p(x)$ the probability that the interactive machine $V$ accepts, on input $x$, when interacting with the prover specified by $P_{x, y, r}$. Now, if $p(x)>\mu(|x|)$ then, on input $x$ and access to oracle $P_{x, y, r}$, with probability at least $1-\mu(|x|)$, machine $K$ outputs a solution $s$ for $x$. That is:

$$
\text { If } p(x)>\mu(|x|) \text {, then } \operatorname{Pr}\left[\left(x, K^{P_{x, y, r}}(x)\right) \in R\right]>1-\mu(|x|) .
$$

The oracle machine $K$ is called a strong knowledge extractor.

An interactive pair $(P, V)$ so that $V$ is a strong knowledge verifier for a relation $R$ and $P$ is a machine satisfying the non-triviality condition (with respect to $V$ and $R$ ) is called a system for strong proofs of knowledge for the relation $R$.

Thus, it is required that whenever $p(x)>\mu(|x|)$ (i.e., whenever the prover convinces the verifier with non-negiligible probability), the extractor fails with negligible probability. Our choice to bound the failure probability of the extractor by the specific negligible function $\mu$ (which serves mainly as bound on $p(x)$ ) is rather arbitrary. What is important is to have this failure probability be a negligible function of $|x|$. Actually, in case membership in the relation $R$ can be determined in polynomial-time, one may reduce the failure probability from $1-\frac{1}{\operatorname{poly}(n)}$ to $2^{- \text {poly }(n)}$, while maintaining the polynomial running-time of the extractor. Finally, we note that the extractor presented in the next section has failure probability 0 . 


\section{On the existence of strong proofs of knowledge}

Some zero-knowledge proof (of knowledge) systems for NP are in fact strong proofs of knowledge. In particular, consider $n$ sequential repetitions of the following basic proof system for the Hamiltonian Cycle ( $\mathrm{HC}$ ) problem (which is NP-complete). We consider directed graphs (and the existence of directed Hamiltonian cycles), and employ a commitment scheme $\left\{C_{n}\right\}$ as above.

Construction 2 (Basic proof system for $\mathrm{HC}$ ):

- Common Input: a directed graph $G=(V, E)$ with $n \stackrel{\text { def }}{=}|V|$.

- Auxiliary Input to Prover: a directed Hamiltonian Cycle, $C \subset E$, in $G$.

- Prover's first step (P1): The prover selects a random permutation, $\pi$, of the vertices of $G$, and commits to the entries of the adjacency matrix of the resulting permuted graph. That is, it sends an $n$-by-n matrix of commitments such that the $(\pi(i), \pi(j))^{\text {th }}$ entry is $C_{n}(1)$ if $(i, j) \in E$, and $C_{n}(0)$ otherwise.

- Verifier's first step (V1): The verifier uniformly selects $\sigma \in\{0,1\}$ and sends it to the prover.

- Prover's second step (P2): If $\sigma=0$, then the prover sends $\pi$ to the verifier along with the revealing (i.e., preimages) of all $n^{2}$ commitments. Otherwise, the prover reveals to the verifier only the commitments to the $n$ entries that correspond to $C$; that is, it reveals the $(\pi(i), \pi(j))^{\text {th }}$ entry if and only if $(i, j) \in C$. (By revealing a commitment $c$, we mean supply a preimage of $c$ under $C_{n}$; i.e., a pair $(\sigma, r)$ so that $c=C_{n}(\sigma, r)$.)

- Verifier's second step (V2): If $\sigma=0$, then the verifier checks that the revealed graph is indeed isomorphic, via $\pi$, to G. Otherwise, the verifier just checks that all revealed values are 1 and that the corresponding entries form a simple $n$-cycle. (Of course in both cases, the verifier checks that the revealed values do fit the commitments.) The verifier accepts if and only if the corresponding condition holds.

The reader may easily verify that sequentially repeating the basic protocol for $n$ times yields a zero-knowledge proof system for HC, with soundness error $2^{-n}$. We argue that the resulting system is also a strong proof of knowledge of the Hamiltonian cycle. Intuitively, the key observation is that each application of the basic proof system results in one of two possible situations depending on the verifier's choice, $\sigma$. In case the prover answers correctly in both cases, we can retrieve an Hamiltonian cycle in the input graph. On the other hand, in case the prover fails in both cases, the verifier will reject regardless of what the prover does from this point on. This observation suggests the following construction of a strong knowledge extractor (where we refer to repeating the basic proof systems $n$ times and set $\left.\mu(n)=2^{-n}\right)$.

Strong knowledge extractor for Hamiltonian cycle: On input $G$ and access to the prover-strategy oracle $P^{*}$, we proceed in $n$ iterations, starting with $i=1$. Initially, $T$ (the transcript so far), is empty. 
1. Obtain the matrix of commitments, $M$, from the prover strategy (i.e., $M \leftarrow$ $\left.P^{*}(T)\right)$.

2. Obtain the prover's answer to both possible verifier moves; that is, for every $\sigma \in\{0,1\}$, obtain the corresponding answer $A_{\sigma} \leftarrow P^{*}(T, \sigma)$. Each of these answers may be correct (i.e., passing the corresponding verifier check) or not.

3. If both answers are correct, then we recover a Hamiltonian cycle. In this case the extractor outputs the cycle and halts.

4. In case a single answer, say the one for value $\sigma$, is correct and $i<n$, we let $T \leftarrow(T, \sigma)$, and proceed to the next iteration (i.e., $i \leftarrow i+1)$. Otherwise, we halt with no output.

Note that we reach iteration $i$ only if and only if in each of the prior $i-1$ iterations a single verifier choice is answered correctly (and we have appended this choice in $T$ ). Hence, if the extractor halts with no output in iteration $i<n$, then the verifier (in the real interaction) accepts with probability zero (since in iteration $i$ both verifier choices yield incorrect answers). Similarly, if the extractor halts with no output in iteration $n$, then the verifier (in the real interaction) accepts with probability at most $2^{-n}$ (since at most one choine is answered correctly). Thus, whenever $p(G)>2^{-n}$, the extractor succeeds in recovering a Hamiltonian cycle (with probability 1 ).

\section{Postscript}

This section was added in the current revision and provides some support for conjectures made explicitly or implicitly in the original text.

Regarding our conjecture that there exist proofs-of-knowledge that are not strong proofs-of-knowledge, partial evidence is provided by subsequent work of Barak, Lindell, and Vadhan [1,2]. Both work refer to constant-round zeroknowledge protocols (for sets outside $\mathcal{B P} \mathcal{P}$ ), and the seperation relies on the existence of such protocols (under standard computational assumptions) that are (ordinary) proofs of knowledge for NP-relations.

1. Barak and Lindell [1] show that such prtotocols cannot have a strict probabilistic polynomial-time black-box extractor, which implies that they cannot be proven to be strong proofs-of-knowledge in a black-box manner. (Still, recall that non-black-box extractors may exist.)

2. Barak, Lindell, and Vadhan [2] show that if (exponentially) strong one-way permutations exist, then such prtotocols cannot have a strict probabilistic polynomial-time extractor, which implies that they cannot be strong proofsof-knowledge.

The existence of constant-round zero-knowledge protocols that are (ordinary) proofs of knowledge for NP-relations can be based on standard intractability assumptions: See Feige and Shamir [5] for the case of argument systems and Lindell [12] for the case of proof systems. 


\section{References}

1. B. Barak and Y. Lindell. Strict Polynomial-Time in Simulation and Extraction. SIAM J. on Comput., Vol. 33 (4), pages 783-818, 2004.

2. B. Barak, Y. Lindell, and S. Vadhan. Lower Bounds for Non-Black-Box ZeroKnowledge. J. of Comp. and Sys. Sci., Vol. 72 (2), pages 321-391, 2006.

3. M. Bellare and O. Goldreich. On Defining Proofs of Knowledge. In Crypto92, Springer-Verlag LNCS (Vol. 740), pages 390-420.

4. U. Feige, A. Fiat and A. Shamir. Zero-Knowledge Proofs of Identity. J. of Crypto., Vol. 1, 1988, pages 77-94.

5. U. Feige and A. Shamir. Zero-Knowledge Proofs of Knowledge in Two Rounds. In Crypto89, Springer-Verlag LNCS (Vol. 435), pages 526-544.

6. O. Goldreich. Secure Multi-Party Computation. Unpublished manuscript, 1998. Available from http://www.wisdom.weizmann.ac.il/ oded/foc.html Superseded by [8, Chap. 7].

7. O. Goldreich. Foundation of Cryptography: Basic Tools. Cambridge University Press, 2001.

8. O. Goldreich. Foundation of Cryptography: Basic Applications. Cambridge University Press, 2004.

9. O. Goldreich. On Expected Probabilistic Polynomial-Time Adversaries - A suggestion for restricted definitions and their benefits. J. of Crypto., Vol. 23 (1), pages $1-36,2010$.

10. O. Goldreich, S. Micali and A. Wigderson. Proofs that Yield Nothing but their Validity or All Languages in NP Have Zero-Knowledge Proof Systems. J. of the $A C M$, Vol. 38, No. 1, pages 691-729, 1991. Preliminary version in 27th FOCS, 1986.

11. S. Goldwasser, S. Micali and C. Rackoff. The Knowledge Complexity of Interactive Proof Systems. SIAM J. on Comput., Vol. 18, pages 186-208, 1989. Preliminary version in 17th STOC, 1985.

12. Y. Lindell. Constant-Round Zero-Knowledge Proofs of Knowledge. Manuscript, 2010.

13. M. Tompa and H. Woll. Random Self-Reducibility and Zero-Knowledge Interactive Proofs of Possession of Information. University of California (San Diego), Computer Science and Engineering Department, Technical Report Number CS92244, June 1992. Preliminary version in 28th FOCS, pages 472-482, 1987. 